\title{
Substances of abuse - demand for their determinations in the Western Cape
}

Pieter van der Bijl, 4th-year medical student

Department of Pharmacology, University of Stellenbosch, Tygerberg

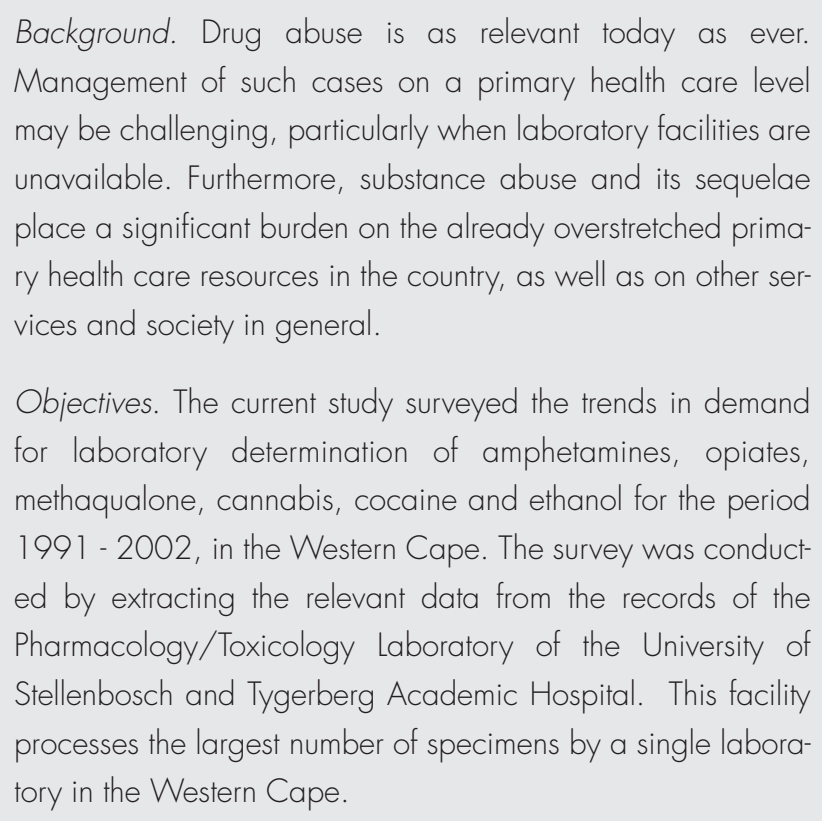

Objectives. The current study surveyed the trends in demand for laboratory determination of amphetamines, opiates, methaqualone, cannabis, cocaine and ethanol for the period 1991 - 2002, in the Western Cape. The survey was conducted by extracting the relevant data from the records of the Pharmacology/Toxicology Laboratory of the University of Stellenbosch and Tygerberg Academic Hospital. This facility processes the largest number of specimens by a single laboratory in the Western Cape.

Results. From the data obtained a seasonal pattern emerged for all substances except ethanol, with a trough appearing in early winter. Demand for ethanol analysis was fairly constant throughout the year, with a peak in the last quarter. Ethanol level was the most frequently requested analysis between 1991 and 1997. This concurs with its status as the main substance of abuse in South Africa and the rest of the world. There was an increased demand for analysis of amphetamines, opiates, methaqualone, cannabis and cocaine between 1991 and 2002. Generally dominating, next to ethanol, were requests for cannabis and methaqualone analysis. Interesting to note was the increase in demand for opiate analysis, following the trend observed in certain other regions of the world.

Conclusion. The analysis trends observed in this study demonstrate global patterns of drug abuse emerging in the Western Cape. The medical and social effects of drug abuse impose a grave responsibility on policymakers to ensure that adequate funding is available for analytical laboratories. Only in such a way can these patients be correctly diagnosed and treated. 
The use and abuse of substances, and the impact on primary health care, are particularly relevant today, also in South Africa. Furthermore, the escalating tendency to abuse a variety of substances simultaneously, particularly ethanol in combination with another substance, often presents diagnostic challenges beyond the capacity of the general practitioner working at primary level without laboratory facilities. In spite of a relative paucity of data, there appears to be an increased use of licit and illicit substances, including ethanol, among teenagers in South Africa. This was revealed by studies conducted among high school pupils in the Cape Peninsula and, more recently, in KwaZulu-Natal. 1,2

Cape Town features very prominently as far as drug abuse and its effects are concerned. Compared with other local sites, it has the highest proportion of ethanol-related psychiatric discharge diagnoses, the highest proportion of ethanol-positive trauma patients injured through violence and traffic-related accidents, the highest proportion of ethanol-positive, unnatural deaths and the highest proportion of arrestees who reported being intoxicated at the time of the alleged offence. ${ }^{3}$ Cape Town also has the highest proportion of trauma patients testing positive for cannabis, Mandrax and cocaine. ${ }^{4}$

Apart from the substantial burden in terms of treatment, health care costs, deaths and drug-related crime, there exists the very real and sinister potential of synergism between drugs of abuse and neurotoxins released during the course of HIV infection. In such cases this may lead, possibly via dopaminergic mechanisms, to accelerated and more severe neurocognitive dysfunction than occurs in HIV populations that do not abuse drugs. ${ }^{5}$

The Pharmacology/Toxicology Laboratory of the University of Stellenbosch and Tygerberg Hospital provides a 24-hour service primarily to the Tygerberg Academic Hospital, and also an afterhours service to Groote Schuur, Victoria, Red Cross, 2 Military and a number of smaller hospitals. Blood and urine samples are also received by the abovementioned facility from several drug/alcohol rehabilitation centres and private pathology laboratories. The Pharmacology/Toxicology Laboratory processes the largest number of specimens by a single laboratory in the Western Cape. The total number of requests received for determining the presence of drugs of abuse and ethanol levels (approximately 9000 in 2002) is therefore probably the best available reflection of the demand for these analyses in the Western Cape, and as a corollary, also a reflection of the trends of drug abuse.

The aim of this study was to survey the trends in demand for laboratory determinations of various substances of abuse over a 12 - year period (1991 - 2002) at the Pharmacology/Toxicology Laboratory of the University of Stellenbosch and Tygerberg Academic Hospital.

\section{Methods}

Laboratory records from the Pharmacology/Toxicology Laboratory were examined, spanning the period 1991 - 2002. The number of requests for urinary determinations of five drugs of abuse, i.e. amphetamines, opiates, methaqualone, cannabis and cocaine, and blood levels of ethanol were extracted from these records. All urinary determinations of the drugs of abuse are performed qualitatively by means of a homogeneous enzyme immunoassay technique, while the ethanol blood levels are measured quantitatively by radiative energy attenuation technology. The data were transferred to an Excel spreadsheet (Microsoft Incorporated, Seattle, USA). Thereafter, the number of requests for determinations performed (totals per month and totals per year between 1991 and 2003) was plotted for each drug of abuse and ethanol using GraphPad Prism software (GraphPad Software Inc, San Diego, USA).

\section{Results}

The number of analyses for amphetamines, opiates, methaqualone, cannabis, cocaine and ethanol for the period 1991 - 2002, requested per month and per year, is shown in Figs 1 and 2, respectively.

\section{Discussion}

Several of the previously mentioned hospitals, other than Tygerberg Academic Hospital, as well as certain private pathology laboratories, provide their own daytime analytical services for drugs of abuse and ethanol, while some of the smaller institutions also use so-called 'near-patient testing devices' (side room tests). However, the latter is an expensive and potentially inaccurate method for determining drug abuse status. ${ }^{6}$ As a result of the other daytime services, data obtained from the current study are therefore necessarily somewhat conservative with regard to the true demand in the Western Cape.

From the number of requests per month (Fig. 1) it appears that there is a trough in early winter, and therefore a seasonal pattern in the demand for amphetamine, opiate, methaqualone, cannabis and cocaine analysis. The demand for ethanol analysis remains fairly constant at approximately 1300 samples per month, but rises to approximately 1500 per month in the last 
quarter of the year, a period coinciding with the summer holidays in South Africa (Fig. 1). While no definite reason can be given for this observation, higher usage of ethanol may be related to longer hours that users/abusers spend socialising in the warmer months during the holiday/festive season. Furthermore, more money may be available for recreational purposes during this time of the year as a result of bonuses.

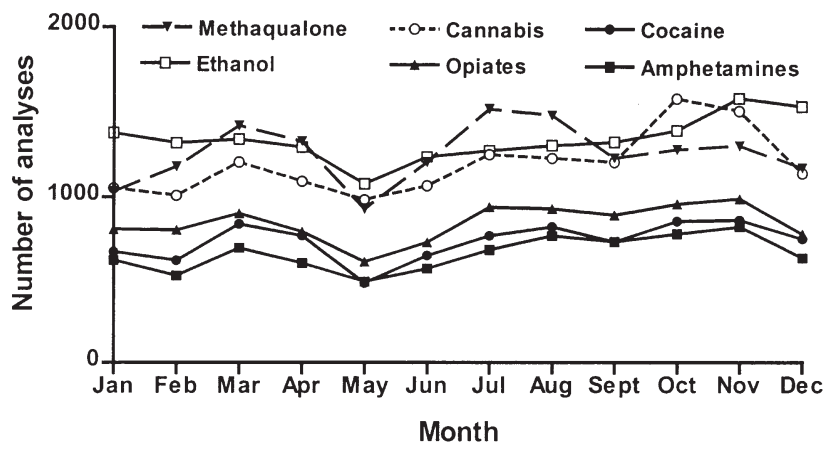

Fig. 1. Total number of analyses for substances of abuse per month (1991 - 2002).

Ethanol blood level determinations were the most frequently requested analyses between 1991 and 1997 (Fig. 2), an observation in agreement with its status as the main substance of abuse in South Africa. ${ }^{7}$ The conclusion may therefore be drawn that the Western Cape is no exception in this regard. After 1997, the demand for ethanol determinations decreased to being lower than determinations for other substances by 2001 .

As far as the other chronological trends are concerned (i.e. aside from ethanol), it is clear from Fig. 2 that there has been a steady increase in the number of requests for analysis of amphetamines, opiates, methaqualone, cannabis and cocaine between 1991 and 2002. Generally dominating (next to ethanol) are requests

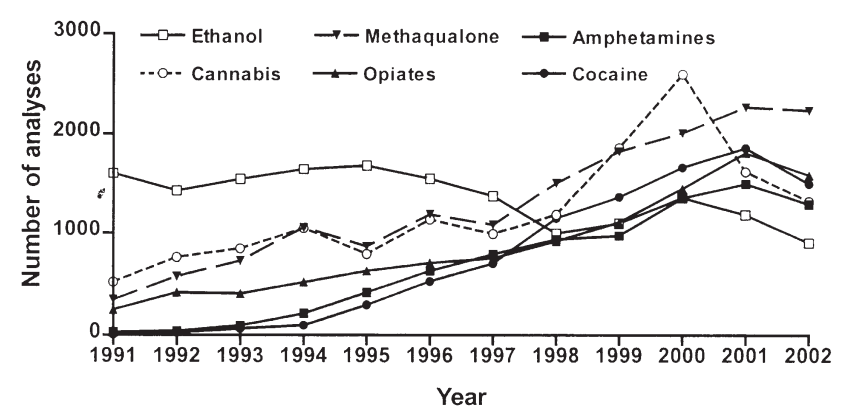

Fig. 2. Total number of analyses for substances of abuse per year (1999 - 2002). for cannabis ('dagga') and methaqualone ('Mandrax') determinations, with total numbers of samples analysed for these two drugs in excess of 2000 in the year 2000. This concurs with published South African data (1997 - 1999) which have demonstrated that cannabis and methaqualone, alone or in combination, are the most frequently reported illicit drugs of abuse. ${ }^{4}$ These two agents are generally responsible for the largest numbers of drug-related police arrests, drug-related psychiatric diagnoses and drug-related trauma in South Africa. ${ }^{4}$ Due to the increased use of cannabis over the last decade in all regions of the world, following a decline in the 1980s, it is by far the most widely abused substance, with estimated consumption rates of at least $2 \%$ among the global population. ${ }^{8}$ The Western Cape therefore follows this trend for the most commonly abused drug. However, the combined use of cannabis and methaqualone (the so-called 'white pipe') appears to be an almost uniquely South African habit.

Worldwide, the popularity of cannabis is followed, according to region, by amphetamine-type stimulants or cocaine. ${ }^{8}$ This pattern is also emerging in the Western Cape, as shown by this survey, with requests for cocaine analysis showing the third fastest growth rate (after cannabis and methaqualone) between 1998 and 2002

In contrast to the overseas data, where opiate abuse was found to be far less widespread, ${ }^{8}$ requests in the present study for opiate analysis were fourth on the list between 1991 and 1997, approximately equalling those for amphetamines between 1998 and 2000. In 2001 and 2002 the demands for opiate analysis competed with cocaine determinations for fourth place on the list. This follows the trend of increasing opiate abuse observed in other regions of the world, e.g. Eastern Europe, Asia and the rest of Africa. ${ }^{8}$

After 2000, there was a decreased demand for cannabis and ethanol analysis, and after 2001, for cocaine, opiate and amphetamine analysis. These changes merely reflect a reduction in the total number of samples sent to the laboratory because one of the private pathology groups, previously one of the major clients, established its own drug monitoring laboratory.

\section{Conclusions}

As far as can be ascertained, this is the first extended trend study with regard to the demand for determination of substances of abuse in the Western Cape. The exact extent to which these trends reflect the national and global situation is unknown, but there appears to be a tendency, reflected by the analytical 
demands, towards general similarity with worldwide substance abuse patterns. This probably has its origin in the increasing globalisation of illicit drug production, trafficking and consumption.

Since substances are abused not only in affluent societies but also in poor communities, they consequently impose a very direct and heavy burden on the already overstretched primary health care resources in South Africa. Only with adequately equipped and funded analytical drug monitoring facilities can those addicted to or overdosed with substances, or in any other way abusing substances, be diagnosed correctly and treated appropriately. Ongoing drug screening of a patient receiving therapy for drug addiction is, at least in the initial phases of treatment, of paramount importance in the success of rehabilitation.

It must be kept in mind that many drug/ethanol abusers are teenagers, who tend to abuse more than one substance simultaneously, e.g. cannabis and ethanol. ${ }^{9}$ Moreover, the latter are 'gateway drugs' which often lead to increased use of more 'potent' drugs such as cocaine. ${ }^{10}$ Considering the widespread medical and social consequences of substance abuse, as well as the short- and long-term sequelae to mental and physical health of acute and chronic abuse, health care policymakers carry a grave responsibility to ensure that budgets for state-funded drug analytical facilities are adequate. This is essential not only for the diagnosis of drug abuse, but also for monitoring the ensuing process of rehabilitation.
I would like to express my gratitude to Mr Jan de Bruyn, Control Technologist, and Mrs Sunelle Hanekom, Senior Secretary, Department of Pharmacology, for their help in extracting the data from the laboratory records. I am also grateful to Professor Pieter van der $\mathrm{Bij}$, Head of the Department of Pharmacology, who encouraged me to undertake this study and made constructive comments on the manuscript.

\section{References}

1. Flisher AJ, Ziervogel CF, Chalton DO, et al. Risk-taking behaviour of Cape Peninsula highschool students. Part V. Drug use. S Afr MedJ 1993; 83: 483-485.

2. Taylor $M$, Jinabhai $C C$, Naidoo $K$, et al. An epidemiological perspective of substance abuse among high school pupils in rural KwaZulu-Natal. S Afr Med J 2003; 93: 136-140.

3. Parry CD, Bhana A, Myers B, et al. Alcohol use in Sou h Africa: Findings from The South African Community Epidemiology Network on Drug Use (SACENDU) Project. I Stud Alcohol 2002; 63: 430-435.

4. Parry CD, Bhana A, Pluddemann A, et al. The Sou h African Community Epidemiology Network on Drug Use (SACENDU): description, findings (1997-99) and policy implications. Addiction 2002; 97: 969-976.

5. Nath A, Hauser KF, Woina V, et al. Molecular basis for interactions of HIV and drugs of abuse. J Acquir Immune Defic Syndr Hum Retrovirol 2002; 31: Suppl 2; S62-69.

6. George S, Bra thwaite RA. Use of on-site testing for drugs of abuse. Clin Chem 2002; 48: $1639-1646$

7. Parry CD. Alcohol and Other Drug Use. South African Health Review 2000. Durban: Health Systems Trust, 2000: 441-454

8. Costa e Silva JA. Evidence-based analysis of the worldwide abuse of licit and illicit drugs. Human Psychopharmacology 2002; 17: 131-140.

9. Flisher AJ, Ziervogel CF, Chalton DO, et al. Risk-taking behaviour of Cape Peninsula highschool students. Part X. Multivariate relationships amongst behaviours. S Afr Med J 1996 86: $1094-1096$

10. World Hea th Organisation. The Health of Young People. A Challenge and a Promise Geneva: World Hea th Organisation, 1993 